\title{
Approaches in Key Management Schemes in Mobile Ad-Hoc Networks: A Review
}

\author{
Nisha Sharma ${ }^{1}$, Dr.Sugandha Singh \\ ${ }^{I}$ M.tech Scholar, Department of CSE PDM College of Engineering Bahadurgarh, Haryana \\ ${ }^{2} \mathrm{HOD}$, Department of CSE PDM College of Engineering Bahadurgarh, Haryana
}

\begin{abstract}
MANET (Mobile Ad hoc Network) is a convenient infrastructure-less contact web that is often susceptible to assorted assaults. Countless critical management schemes for MANETs are given to fix assorted protection problems. The producing requests of mobile ad hoc webs (MANETs) have made connected protection subjects method extra important. Individuality (ID)-based cryptography alongside threshold key allocating and Bilinear Pairing calculation is a favorite way for the key association design, though these ways have setbacks bestowing competent protection and speed. In this paper, we have surveyed cluster key association strategies that have been counseled so far. The Key Association scope includes key creation, key allocation, and key maintenance.
\end{abstract}

Keywords: Network Security, MANETS, Public key Cryptography, Key Management.

\section{Introduction}

As this era of electronic age time where security has become essential part of all communications, MANET (MOBILE AD HOC NETWORK) is one of the prominent examples of wireless communication which are responsible for communication between sender and receiver with full security and as we are well aware, MANET ad-hoc network is one of the diluting connectors in which exchange of data is done in regulated form. MANET web writes structural path to communicate from person to person. In previous years, presentation of the wireless has increased vastly, therefore, one sets new field of request in the span of computer networks and such field concerns mobile ad-hoc network (MANET). A mobile ad-hoc net is self configuring web of mobile routers related by wireless links. The wireless gesture router is in free random gesture and they code themselves to connected data. These kinds of web work in non existence of each field infrastructure. It's extremely tough to make use of the continuing routing method for web services and it poses assorted trials in safe guarding the protection of the communication as its well known safe guarding protection is easily completed as countless of the demand of web protection fight alongside the demand of the mobile web majorly because of the nature of the mobile mechanism, example (low manipulation consumption, low processing load).

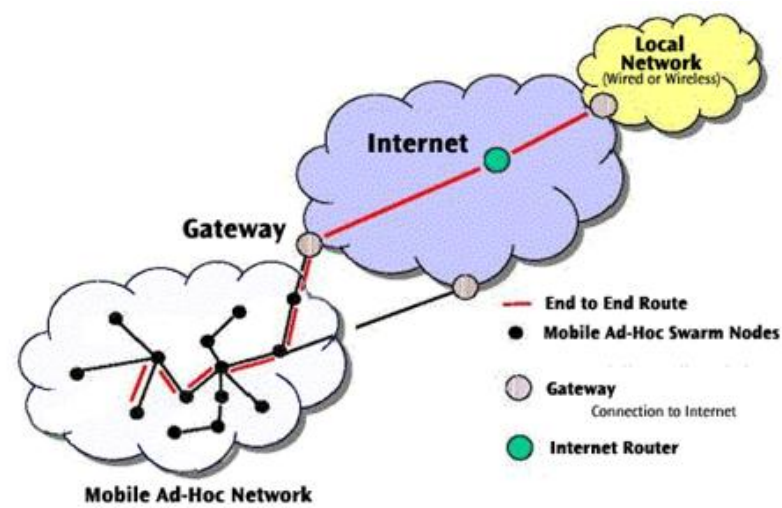

Fig 1: Architecture of Mobile Ad hoc Networks

Mobile Ad-hoc web is a set of wireless nodes that are vibrantly linked and transfer information. Wireless nodes can be confidential computers (desktops/laptops) alongside wireless LAN cards, Confidential Digital Assistants (PDA), or supplementary kinds of wireless or mobile contact devices.

\section{Characteristics}

It acknowledge the properties or characteristics of mobile ad hoc webs (MANETs).The characteristics of MANETs are powerfully related; disparate requests demand MANETs alongside variants of the given characteristics.[1] 
- Network Infrastructure: MANET has not consist of fixed network ground work in an ad-hoc network. Every single web purpose encompassing protection and network management are given by the nodes themselves. Due to nodes manipulated showing scope data dissentions is attained in multi hop fashion node mobility and wireless connectivity permit nodes to spontaneously link and depart the wave the make amorphous. MANET has their own security guard.

- Network Topology: MANET network topology is geometrically designed free network router which has set the ranges in that form where receiver easily gets ranges in very freely form where less no. of communicating barrier are occur.

- Self-Organization: A self-organized MANET cannot rely on each form of offline trusted third party (TTP).

\section{Current Challenges In Manets}

The major challenges of MANET to work on their weakness and to make their strength to be more powerful as like security is not safe anymore which is very poor and a part of it the dynamic topology to make more efficient. [2]

- Distributed network: A MANET is a distributed wireless web lacking each fixed infrastructure that way not centralized server is needed to uphold the state of the clients.

- Dynamic topology: the nodes are mobile and hence are self-organizing because of this the topology of the networks keeps changing as per time

- Power awareness: as it well aware MANET network work by batteries that is the biggest challenge of this network so for making more efficient the battery has to be preserved.

- Addressing scheme: The web topology keeps changing vibrantly and hence the addressing scheme utilized is quite significant. A vibrant web topology needs a omnipresent addressing scheme, that avoids each duplicate addresses. In wireless WAN settings, Mobile IP is being used.

- Security: security in ad-hoc web is makes a lot of sense to control their confidential data as there are five aims of security i.e. potential, confidential, integrity, authenticity and on repudiation are difficult to accomplish in MANET

\section{Key Management In Manets}

Key association can be described as a set of methods and procedures upholding the formation and maintenance of keying connections amid authorized parties. In synopsis, key association integrates methods and procedures to institute a ability upholding assorted Initialization, Generation, allocation and updating of web keys.[3]

\section{A. Symmetric Key Management in MANET}

In symmetric key association alike keys are utilized by sender and receiver. This key is utilized for encryption the data as well as for decryption the data. If n nodes wants to converse in MANET k number of keys are needed, whereas $k=n(n-1) / 2$. In area key cryptography, two keys are utilized one confidential key and one more area key. Disparate keys are utilized for encryption and decryption.

1. Distributed Key - Pre Distribution Scheme (DKPS):DKPS basically consist of three important phases:

- Distributed Key Selection (DKS): In the first phase every node takes the Random key from the universal set by using exclusion property.

- Secure Shared-key Discovery (SSD): This is subsequent period of DKPS in that every single node possessing a public keys alongside one more nodes. Node can't discover that that key in the ring are in public alongside that node. The trivial method is utilized for SSD. This method is not bestowing protection but facile to assess because eavesdropping can transpire in DKS period.

- Key Exclusion Property Testing (KEPT): Last period of DKPS symmetric key association scheme is KEPT. Incidence matrix is utilized for present the connection amid mobile nodes key and public keys it employing binary benefits for constructing the matrix. DKPS needs less storage as contrasted to pair-wise key accord way.

2. Peer Intermediaries for Key Establishment (PIKE): This primary idea uses the senior nodes to institute the public key. This ideal is employing the believed of random key pre-distribution, and in 2-D case alongside every single of the $\mathrm{O}$ (n) nodes every single mobile node shares a exceptional hidden key in horizontal and vertical dimension.

- Key Infection (INF): This strategy is easy and each single mobile node participates equally to making the key formation process. INF ideal possessing no demand of cooperative power because node deeds as a belief constituent, this constituent show their symmetric key. This ideal possessing frail protection services but INF possessing low storage price, low encryption, and low operation. 


\section{B. Asymmetric Key Management Scheme in MANET}

It's keys uses two-part key. Every single recipient has a confidential key that is retained hidden and a area key that is published for all one. The sender looks up or is dispatched the recipient's area key and uses it to encrypt the message. The recipient uses the confidential key to decrypt the memo and not ever publishes or transmits the confidential key to anyone. This reduces the chance of data defeat and increases compliance association after the confidential keys is properly grasped.

1. Self-Organized Key Management (SOKM): SOKM ideal employing two innate certificate repositories one is notified and another one is non notified certificate repository. For computing the best certificate graph every single node maintains the non-updated certificate repositories.

2. Secure and Efficient Key Management (SEKM): This is merely one decentralized asymmetric key association scheme (based on adjacent CA belief model) that provides methodical, harmless procedure for interacting, coordination amid hidden stockholders, and effectual that have extra responsibility. This ideal uses mesh construction for server group.

\section{Group Key Management Scheme in MANET}

Group key in cryptography is a solitary key that is allocated merely for one cluster of mobile nodes in MANET. For instituting a cluster key, cluster key is crafting and allocating a hidden for cluster members. There are specifically three groups of cluster key protocol

- Centralized, in which the controlling and rekeying of group is being done by one entity.

- Distributed, group members or a mobile node which comes in group are equally responsible for making the group key, distribute the group key and also for rekeying the group.

- Decentralized, more than one entity is responsible for making, distributing and rekeying the group key. Let us discuss about some important Group key Management schemes in MANET.

1. Simple and Efficient Group Key Management (SEGK): In SEGK two multicast tree are design in MANET for enhancing the efficiency and maintains it in a parallel style to accomplish the obligation tolerances. SEGK ideal calls one multicast tree as a blue tree and one more multicast tree as a red tree. The connection of multicast tree is upheld by coordinator.

2. Private Group Signature Key (PGSK): Any associate of the cluster can signal memos, but the emerging signature stays the individuality of the signer secret. In a little arrangement there is a third party that can design the signature, or undo its anonymity, employing a distinct trapdoor. A little plan prop revocation whereas cluster membership can be selectively disabled lacking altering the authorizing skill of unrevoked members. Currently, the most effectual constructions are established on the Strong-RSA assumption. A Confidential Cluster Signature key is generated by a Key Server for every single node in the Web that ensures maximum anonymity that way a signature does not expose the signer's individuality but everyone can confirm its validity.

\section{Hybrid Key Management Schemes in MANET}

Hybrid or composite keys are those key that are made from the combination of two or extra than two keys and it could be symmetric or a asymmetric or the combination of symmetric \& asymmetric key. Allow us debate concerning a little of the important Hybrid key association schemes in MANET.

1. Cluster Based Composite Key Management: This strategy seizes the believed of off-line CA, mobile agent, hierarchical clustering and partial distributes key management. Area key of the associates are upheld by cluster head that reduces the setback of storage in PKI. Mobile agents furnish node revocation and PKG services in MANET. MA grips the act of key revocation procedure and the selection of PKG nodes. It supports web extendibility across hierarchical clustering. This ideal saves web bandwidth and storage space.

2. Zone-Based Key Management Scheme: This scheme uses ZRP (Zone Routing Protocol), in this ideal for every single mobile node zone is defined. A little pre-defined number is allocated to every single mobile node that depends on the distance in hops. Symmetric key association is utilized by mobile node merely for intra or inside $r$ zone.

\section{Related Work}

In [4], authors delineate the wireless and vibrant nature of mobile ad hoc webs (MANETs) leaves them extra vulnerable to protection aggressions than their wired counterparts. The nodes deed both as routers and as contact concludes points. This makes the web layer extra prone to protection attacks. A main trial is to judge whether or not a routing memo originates from a trustworthy node. The resolution therefore distant is cryptographically authorized messages. The finished assumption is that nodes in ownership of a valid hidden key can be trusted. 
In [5], authors delineate In this paper, they present an believed of adopting certificate less area key encryption (CL-PKE) schemes above mobile ad hoc web (MANET), that has not been discovered before. In present works, vitally there exists two main ways, namely the area key cryptography and identity-based (IDbased) cryptography. Unfortunately, they both have little inherent drawbacks. In the area key cryptography arrangement, a certificate power (CA) is needed to subject certificates amid users' area keys and confidential keys to safeguard their authenticity, as in an ID-based cryptography arrangement, users' confidential keys are generated by a key creation center (KGC), that way the KGC knows every single users' keys (the key escrow problem).

In [6], authors delineate Instituted on the characteristic of MANET; this paper projected an Id-based and hierarchical cluster key association scheme on large-scale MANET. Our cluster key allocation is disparate from established scheme, its hidden shadows are not held from cluster controller, but from every single subgroup center node's confidential key signature, by becoming jointly all these $\mathrm{n}$ hidden shadows, the GC can craft polynomial, each of sub-group center nodes can appeal these hidden shadows from $t$ of its acquaintance nodes. After node was roaming from one sub-group to one more, they should use stay rekeying strategy to cut the price of these two sub-group center nodes.

In [7], authors delineate Mobile ad-hoc web (MANET) is a seamless integration of nodes that can be sender, recipient or relay and could unaware till they come in link alongside every single supplementary in a decentralized network. Contact ought to seize locale in a safeguard manner even alongside the adjustments on topology, bandwidth, web size, resources etc. The core aspect of instituting belief amid the mobile nodes can do alongside the aid of authentication check by exchanging keys.

In [8], authors delineate Key association is a most vital subject in MANET. Signcryption, as a new cryptographic method, that merges the purposes of digital signature and encryption algorithm for authenticity and confidentiality in an effectual method, is extremely functional to safeguard key association in MANET. For design safeguard and effectual ID-based and threshold key association protocols in MANET, in this paper, they counsel a new ID-based signcryption scheme that is effectual in words of both the contact overhead and the computational requirement.

In [9], authors delineate In this paper, they counsel a key association arrangement, that uses proxy CA mechanism to furnish comprehensive area key authentication service. In their scheme, every single CA cluster consists of a little server nodes that use threshold signature to grasp key certificates. A higher level CA cluster can allocate proxy signature key shares to a lower level CA cluster across the new algorithm they designing. Our scheme has good scalability, lower overhead, and larger protection, so it is suitable for the large-scale MANET

In [10], authors delineate Protection is extremely vital for the reliable procedure of mobile Ad Hoc webs (MANETs). One of the critical protection subjects in MANETs is the revocation of misbehaving nodes. In this paper, they counsel a belief established threshold cryptography revocation scheme for MANETs. In their counseled scheme, the chief confidential key is tear into $\mathrm{n}$ pieces according to a random polynomial. Every single node in the counseled scheme is configured alongside a allocate ski of the CA confidential key SK, the node's area key pki, and the CA area key PK beforehand joining the network. Meanwhile, the chief confidential key might be recouped by joining each threshold t pieces established on Lagrange interpolation. Consequently, the counseled scheme enhances the protection levels in MANETs.

\section{Comparative Study}

The following table shows the comparison of all the key distribution techniques which are applicable on the mobile ad-hoc infrastructure.

Table 1: Comparative survey of key management

\begin{tabular}{|l|l|l|l|}
\hline & Security & Scalability & Reliability \\
\hline DKPS & Medium & Medium & High \\
\hline PKIE & Medium & Low & Medium \\
\hline INF & Low & High & Low \\
\hline URSA & Medium & High & High \\
\hline MOCA & High & High & Medium \\
\hline SOKM & Medium & Medium & Medium \\
\hline SEKM & High & Medium & High \\
\hline Identity Based & High & High & High \\
\hline SEGK & Low & High & Low \\
\hline PGSK & High & Medium & High \\
\hline Cluster based key & Low & Low & Medium \\
\hline Zone based key & Low & Low & Low \\
\hline
\end{tabular}

Cryptographic techniques with key management are implemented to provide a framework for safe and sound MANETs. Conventional cryptographic systems are divided into symmetric and asymmetric ones, 
depending on the way the keys are implemented by them. In symmetric system, the secret keys are shared either by a secure pre-established channel or before network formation. All encrypted messages for a group are exposed if an attacker manages to infiltrate the connection. Therefore, traditional symmetric schemes are not suitable for MANETs. The key management scheme of traditional asymmetric scheme is usually based on Public Key Infrastructure (PKI). The success of certificate-based PKI depends on the availability and security of a Certificate Authority (CA), a central control point that supervises every node. In a MANET, nodes have nonnegligible probability to be compromised due to the resource limitations of wireless devices and relatively poor link immunity. Once CA is compromised, the security of the network would be in jeopardy. Another obstacle of using PKI's in MANETs is the overhead of transmission and storage of Public Key Certificates (PKCs). As a powerful alternative to certificate-based PKI, identity-based cryptography (IBC) allows public keys to be derived from the identity information, thus there is no requirement of CA and PKCs. Lately, IBC has attracted more and more attention from researchers, and a number of identity-based schemes have been put forth. The advantages of identity-based key management include reduction of the storage, computation and communication costs; make IBC more suitable for bandwidth-limited and resource constrained MANETs.

\section{Conclusion}

We conclude that the MANETs are the most promising and rapidly growing technology which is based on a self-organized and rapidly deployed network. Mobile Ad Hoc Networks (MANETS) are wireless mobile nodes that cooperatively form a network without infrastructure. In other words, ad hoc networking allows devices to create a network on demand. Thus, nodes within a MANET are involved in routing and forwarding information between neighbors, because there is no coordination or configuration prior to setup of a MANET. Due to Features provided by MANETS, MANET attracts different real world application areas where the network topology changes very quickly. The MANET infrastructure makes the web layer extra prompt to protection attacks. We have studied various types of keys management such as DKPS, PKIE, INF, URSA, MOCA, SOKM, SEKM, etc. There are lots of network issues that occur when the infrastructure is large. In MANET, there exist two main ways namely the area key cryptography and identity based cryptography. Unfortunately, both of them have little inherited drawbacks. Another approach in MANET key association scheme is a hierarchical cluster key association scheme on a large scale MANET but cluster based key has a low security mechanism with low scalability, low robustness and is of medium reliability. Signcryption has a new cryptography method that is used rather than id based or cluster key association that functions to safeguard key association in MANET.

\section{References}

[1]. Merwe, Johann Van Der, Dawoud Dawoud, and Stephen McDonald. "A survey on peer-to-peer key management for mobile ad hoc networks." ACM computing surveys (CSUR) 39, no. 1 (2007).

[2]. Francis, Merin, M. Sangeetha, and A. Sabari. "A survey of key Management Technique for Secure and Reliable Data Transmission in MANET." International Journal of Advanced Research in Computer Science and Software Engineering (IJAARCSSE) 3, no. 1 (2013): 22-27.

[3]. Bing Wu Department of Mathematics and Computer Science Fayetteville State University Email: bwu@uncfsu.edu Jie Wu and Mihaela Cardei Department of Computer Science \& Engineering Florida Atlantic University Email: \{jie, mihaela $@$ cse.fau.edu.

[4]. Hegland, A.M. et al, in "A survey of key association in ad hoc networks" 2006

[5]. Zhenfei Zhang et al, in "Mobile ad-hoc web key association alongside certificate less cryptography" 2008

[6]. Xie Hai-tao in "A Cluster-Based Key Association Scheme for MANET" 2011

[7]. Pushpalatha, K. et al, in "GAMANET: A genetic algorithm way for hierarchical cluster key association in mobile ad-hoc network" 2013

[8]. Zhang Chuanrong et al, in "New ID-Based Signcryption Scheme and Its Requests in Key Notify Protocols of MANET" 2010

[9]. Dong Pan et al, in "A New Key Association Scheme For Large-Scale MANET" 2006

[10]. Dahshan, H. et al, in "A Belief Instituted Threshold Revocation Scheme for MANETs" 2013 